\title{
Research on Speech Processing Strategies for English Speakers During Learning of Chinese Quantifier Complements
}

\author{
Jiehua Zhang ${ }^{1}$ and Ruifeng Wang,** \\ ${ }^{1}$ School Office, Shanghai LiDa University, Shanghai, 201609, China \\ ${ }^{2}$ Academic Affairs Office, Beijing Language and Culture University, Beijing, 100083, China \\ *Corresponding author. Email: wangruifeng@blcu.edu.cn
}

\begin{abstract}
This study explored the speech processing strategies for English speakers of three Chinese proficiency levels to learn five types of Chinese quantifier complement sentences through the compiled examination. English speakers experience four speech processing strategies from the low level to the high level when they are processing word order of quantifier complements: sequential combination strategy of verb-quantifiers < verbobject phrase adding strategy < verb-object phrase separation and insertion strategy < separable word separation and insertion strategy. This process reflects the development from external processing to internal processing, that is, from the external adding of components to internal separation and insertion of components. The internal separation of phrases is also manifested by development features from separation among different components to separation of single component.
\end{abstract}

Keywords: second language acquisition; Chinese quantifier complements; speech processing strategies

\section{INTRODUCTION}

Many studies[1-5]on second language acquisition (SLA) of German, English, Japanese and Chinese all found that the second language learners would adopt corresponding speech processing strategies in different stages. There are implicational relations among these speech processing strategies. For example, there are implicational relations in speech processing strategies during word order processing in German learning: "canonical order < adverb preposing < verb separation < inversion < verb-end". This implies that learners must have mastered the left processing strategies (the lower level of processing strategies) of the equation if they have mastered the right processing strategies (the higher level of processing strategies).

There are "verb+ quantifier phrases" in Chinese. For example, “学一个小时” expresses the duration of the learning action. For the internal grammatical relations, the academic circle proposes the verb-object concept[6] and verb-complement concept[7]. The later one is often applied in most Chinese textbooks and grammar books for Chinese teaching. The "quantifier phrases" is taught as "quantifier complements". This language point is the key and challenge in foreigners-oriented Chinese teaching. In particular, the order of verbs, quantifier complements and objects might vary when different verbs or objects are used. Hence, overseas students many make a lot of order errors during acquisition. Scholars[8-10] generally analyze acquisition of quantifier complements from the perspective of errors types or acquisition order. According to associated literature review, no studies have discussed speech processing strategies of quantifier complements yet. In this study, speech processing strategies of quantifier complements for English speakers of the three levels were explored.

\section{RESEARCH DESIGN}

\subsection{Selection of respondents}

English speakers of three Chinese proficiency levels who were learning Chinese were chosen as the respondents. Since all respondents were overseas students who were learning Chinese in a University in China, general information of respondents in grade, learned courses of Chinese, vocabularies of textbooks and the covered language points are explicit. Therefore, operational definition of language level of respondents was proposed mainly according to the Chinese learning hours. Basic conditions of language level grouping are listed as follows.

Table 1. Basic conditions of three levels groups

\begin{tabular}{|c|c|c|c|c|}
\hline $\begin{array}{c}\text { Language } \\
\text { proficiency }\end{array}$ & Grade & $\begin{array}{c}\text { Learned } \\
\text { courses }\end{array}$ & Vocabularies & $\begin{array}{c}\text { Number of } \\
\text { respondents }\end{array}$ \\
\hline $\begin{array}{c}\text { Primary-level } \\
\text { group }\end{array}$ & $\begin{array}{c}1^{\text {st }} \text { Grade } \\
\text { (the second } \\
\text { semester })\end{array}$ & $300-600$ & $1200-2000$ & 20 \\
\hline $\begin{array}{c}\text { Middle-level } \\
\text { group }\end{array}$ & $2^{\text {nd }}$ Grade & $700-1200$ & $2400-4000$ & 20 \\
\hline High-level group & $\geqslant 3^{\text {rd }}$ Grade & $\geqslant 1300$ & $\geqslant 4500$ & 20 \\
\hline
\end{tabular}


The main language points of Chinese, including quantifier complements, have been learned in the first semester of the $1^{\text {st }}$ Grade. In other words, even the primary-level group has learned quantifier complements.

\subsection{Examination design}

The quantifier complements which are invested in this study as well as their sentence types can be divided into following five types:

Type 1: subject + verb + quantifier complements $(\mathrm{S}+\mathrm{V}+\mathrm{O})$. For example, “他跑了一个小时。”

Type 2: subject + verb + quantifier complements + objects $(\mathrm{S}+\mathrm{V}+\mathrm{N}+\mathrm{O})$. For example, “我当了三年兵。”

Type 3: subject + verb + object (specified) +quantifier complements $\left(\mathrm{S}+\mathrm{V}+\mathrm{O}_{\text {specified }}+\mathrm{N}\right)$. For example, “大卫等了 你一个多小时。”

Type 4: subject + verb + object + verb + quantifier complements $(\mathrm{S}+\mathrm{V}+\mathrm{O}+\mathrm{V}+\mathrm{N})$. For example, “我找这本书 找了好几天。”

Type 5: subject + separable word $V_{1}+$ quantifier complements+ separable word $\mathrm{V}_{2}\left(\mathrm{~S}+\mathrm{V}_{1}+\mathrm{N}+\mathrm{V}_{2}\right)$. For example: “我游了一个小时泳。”

Questions in the examination are designed in making sentences with words, which is to put the given words in an order to make a complete sentence. Each type has 6 questions and the whole examination covers 30 questions. Most words for questions in the examination are chosen from Class-A words in the Chinese proficiency test (HSK), and some were Class-B words which were provided with corresponding translations and explanations. All respondents were asked to finish the examination in one hour independently.

\subsection{Research methodology}

The research method which is widely applied in studying speech processing strategies is applied. In other words, the quality and quantity of the learned language points were analyzed by the compiled examination, which were used to analyze the speech processing strategies. Firstly, quantity of accurate sentences in each type of every respondent and number of respondents are input into the database for summary (it is evaluated that respondents who answered 5 of 6 questions of each type accurately have mastered this level). Secondly, testing results are sequenced by an implicational scale to identify the implicational relations among acquisitions of five sentence types. Moreover, index coefficients of the implicational scale are calculated to test validity of the implicational relations. Thirdly, a cluster analysis on acquisition of five sentence types is carried out by using SPSS according to the quantity of respondents and accuracy, thus dividing the acquisition stages. Finally, speech processing strategies that respondents are adopted are analyzed according to sentence acquisition stages of respondents, structure types and relevant statistical data.

\section{EXPERIMENT AND DATA ANALYSIS}

\subsection{Acquisition stages and acquisition sequence of five sentence types}

According to cluster analysis results, three groups achieved basically consistent results in sentence acquisition (Table 2).

Table 2. Chinese quantifier complement acquisition stages of three groups of English speakers

\begin{tabular}{|c|c|c|c|}
\hline $\begin{array}{c}\text { Acquisition } \\
\text { stages }\end{array}$ & $\begin{array}{c}\text { Primary } \\
\text { level }\end{array}$ & $\begin{array}{c}\text { Middle } \\
\text { level }\end{array}$ & High level \\
\hline Stage 1 & $\begin{array}{c}\text { Type 1 } \\
\text { Type 3 }\end{array}$ & $\begin{array}{c}\text { Type 1 } \\
\text { Type 3 }\end{array}$ & $\begin{array}{c}\text { Type 1 } \\
\text { Type 3 } \\
\text { Type 4 }\end{array}$ \\
\hline Stage 2 & $\begin{array}{c}\text { Type 4 } \\
\text { Type 2 }\end{array}$ & $\begin{array}{c}\text { Type 4 } \\
\text { Type 2 }\end{array}$ & Type 2 \\
\hline Stage 3 & Type 5 & Type 5 & Type 5 \\
\hline
\end{tabular}

Three groups had two commons in the acquisition sequence and acquisition stages of quantifier complements. Firstly, they all experienced three stages. Secondly, they showed completely consistent acquisition sequences of five sentence types. Specifically, the primary-level group and middle-level group showed completely consistent acquisition stages and sentence types in each stage. However, high-level group had a small difference in the acquisition stages and Type 4 belonged to Stage 1 .

\subsection{Implicational relations of five sentence types of three groups}

According to testing results, the measurability coefficient of the implicational scale was 0.36 for the primary-level group and 0.27 for the middle-level group, which were lower than the critical value (0.60). This indicated that the implicational scale reflected some implicational relations. However, these implicational relations were weak and couldn't be used to predict acquisition conditions of respondents. The measurability coefficient of the implicational scale was 0.79 for the high-level group, which was higher than the critical value. This reflected that there were significant implicational relations which could be used as references to predict quantifier complement acquisition of the high-level group. Therefore, the acquisition achievements of the high-level group were used to analyze speech processing strategies for quantifier complements. 


\section{ANALYSIS OF SPEECH PROCESSING STRATEGIES}

\subsection{Four speech processing strategies}

The speech processing strategies were analyzed comprehensively according to acquisition sequence, acquisition stages and structural types of each sentence type of the high-level group. Respondents had experienced four speech processing strategies from the low level to the high-level during word order processing of quantifier complements.

\subsubsection{Sequential combination strategy of verb- quantifiers}

all respondents could learn the sequential combination of verbs and quantifier complements easily. They could put quantifier complements accurately after the verbs. Specifically, all respondents had mastered type 1 and 95\% of respondents passed the examination. Type 1 only involves the sequential combination of verbs and quantifier complements except for the subject and basically all respondents had mastered it.

\subsubsection{Verb-object phrase adding strategy}

when there's an object in the quantifier complement sentence, learners combine the verb and object firstly into a phrase and then add the quantifier complements to the phrase. Such external adding methods can be divided into two types. Firstly, quantifier complements are added behind or in front of the verb + object $(\mathrm{V}+\mathrm{O})$ structure, which is mainly manifested by the fact that basically all respondents had learned Type 3 and the passing rate reached $91 \%$. The rule of type 3 was to add quantifier complements behind the $\mathrm{V}+\mathrm{O}$ structure directly. In addition to this structure, respondents also output other sentence structures. However, only few respondents made some sentence structures accurately, which might be caused by a momentary oversight or other occasional factors. These sentence structures were useless in analysis. Key attentions were paid to the speech processing strategies which contributed the structural types of most respondents (structural types that at least 4 respondents had mastered were used as the key analysis objects. Each group had 20 respondents and 4 respondents accounted for at least $20 \%$ ). For Type 3, the structural type which was output accurately by most respondents was only

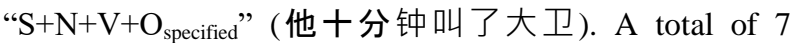
respondents $(35 \%)$ output 11 sentences of Type 3 . This is because respondents adopted the verb-object phrase adding strategy but adding the quantifier complements in front of the $\mathrm{V}+\mathrm{O}$ structure.
Secondly, quantifier complements are added behind the $\mathrm{V}+\mathrm{O}$ structure. A total of $90 \%$ respondents mastered the Type 4. Type 4 is composed of two parts: " $\mathrm{S}+\mathrm{V}+\mathrm{O}$ " and "V+N". Respondents combined the verb and object firstly, and then added the quantifier complements behind the $\mathrm{V}+\mathrm{O}$ structure. For Type 4, respondents output 12 structures and less than $20 \%$ of respondents output each structure accurately. Although such data has no value for analysis, it might find after careful observation that most of these structures had "V+V+O" structures and many respondents mistakenly took two verbs as an overlapping structure. However, "V+V+O" still belonged to the $\mathrm{V}+\mathrm{O}$ structure. Respondents added quantifier complements in front of or behind the $\mathrm{V}+\mathrm{O}$ structure, forming “ $\mathrm{S}+\mathrm{V}+\mathrm{V}+\mathrm{O}+\mathrm{N} ”$ (这本书他们找了找好几天), “ $\mathrm{S}+\mathrm{N}+\mathrm{V}+\mathrm{V}+\mathrm{O}$ ” (我们吃了吃方便面一个星期), “N $+\mathrm{S}+\mathrm{V}+\mathrm{V}+\mathrm{O} ”$ (一下午妈妈洗了洗衣服). Obviously, respondents output these structures by using the verbobject phrase adding strategy. These structural types were more evident in sentences output by the primary-level group and middle-level group, manifested by the high quantity of accurate sentences and the high proportion of respondents who output the sentences accurately.

\subsubsection{Verb-object phrase separation and insertion strategy}

respondents separated the $\mathrm{V}+\mathrm{O}$ structure and insert the quantifier complements between the verb and the object. According to analysis of the verb-object phrase adding strategy, respondents preferred to combine verb and object firstly during sequential combination of verb, object and quantifier complements. In other words, they combined the verb and object into one phrase firstly and then added quantifier complements into the phrase. For Type 2, respondents have to separate the $\mathrm{V}+\mathrm{O}$ structure and then insert the quantifier complements. Hence, respondents have to master such separation and insertion strategy in order to output the sentence accurately. According to acquisition situations, $70 \%$ of respondents mastered the Type 2, indicating that they have mastered the verb-object phrase separation and insertion strategy, but $30 \%$ of respondents haven't mastered the strategy. For questions of Type 2, respondents performed well in " $\mathrm{S}+\mathrm{V}+\mathrm{O}+\mathrm{N}$ " (马克学了汉 语三年) and 7 respondents (35\%) output 16 sentences of type 2 . This was attributed to the use of the verb-object phrase adding strategy which adds quantifier complements after the $\mathrm{V}+\mathrm{O}$ structure.

\subsubsection{Separable word separation and insertion strategy}

Respondents separate the separable words and insert quantifier complements between them. Both verb-object phrase separation and insertion strategy and separable word 
separation and insertion strategy involve separation, the former one separates the $\mathrm{V}+\mathrm{O}$ structure, while the later one separates the separable words which can be used as an integral component. Compared to the $\mathrm{V}+\mathrm{O}$ structure, the separable words are combined more closely. According to acquisition conditions, respondents are difficult to separate the separable words. However, they have to master such separation strategy to learn Type 5. Only 30\% of respondents mastered the Type 5. Specifically, most respondents output “ $\mathrm{S}+\mathrm{V}_{1}+\mathrm{V}_{2}+\mathrm{N}$ ” (学生游泳了一上午) and “ $\mathrm{S}+\mathrm{N}+\mathrm{V}_{1}+\mathrm{V} 2$ ” (她每天一个小时洗澡), because they combined separable words as a verb. Essentially, they adopted the first processing strategy: sequential combination strategy of quantifiers.

To sum up, respondents had to experience four speech processing strategies from the low level to the high level when learning five types of quantifier complements. Firstly, "sequential combination strategy of verbquantifiers" was formed, because it was a relatively simple strategy that only requires determination of the sequence of the verb and quantifier complements. Secondly, the "verbobject phrase adding strategy" was developed. Respondents combined the verb and object into a structure firstly and then added quantifier complements into this structure. Such external adding can be divided into two types. In the first type, quantifier complements are added behind or in front of the $\mathrm{V}+\mathrm{O}$ structure. In the second type, quantifier complements are added behind the $\mathrm{V}+\mathrm{O}$ structure. Thirdly, the "verb-object phrase separation and insertion strategy" was developed. Respondents separated the $\mathrm{V}+\mathrm{O}$ structure and inserted the quantifier complements into the structure. Finally, the "separable word separation and insertion strategy" was developed. Respondents separated the separable words and inserted the quantifier complements into the structure. The development of speech processing strategies of directional complements could be expressed in the following equation:

Sequential combination strategy of verb-quantifiers<verbobject phrase adding strategy<verb-object phrase separation and insertion strategy<separable word separation and insertion strategy.

\subsection{Difficulties in development of speech processing strategies}

If the proportion of respondents who failed to master speech processing strategies was used as the height, the development of speech processing strategies of directional complements can be expressed vividly in the following diagram.

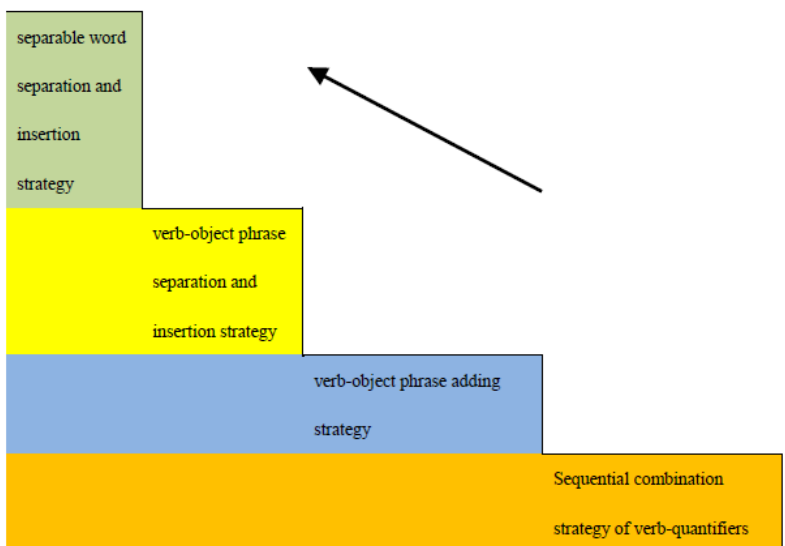

Figure 1. Speech processing strategies of quantifier complements and development layers

In Fig.1, four speech processing strategies can be viewed as four "steps" and the "arrow" implies the development direction of speech processing strategies. To learn the word order of quantifier complements, respondents have to cross over these four "steps" with different amplitudes from the low to high. Respondents climbed the first "step" which was easy to cross over, indicating that all respondents can master the sequential combination strategy of quantifiers easily. Secondly, respondents crossed to the second "step", which was as easy as the first "step" for most respondents. Later, respondents climbed to the third "step", which was relatively more difficult compared to the previous two steps. Finally, respondents climbed to the fourth "step" which was the "highest" and most difficult. Only few respondents could climb onto this "step".

Table 1. Proportions of primary-level group, middlelevel group and high-level group

\begin{tabular}{|c|c|c|c|}
\hline $\begin{array}{c}\text { Sentence } \\
\text { type }\end{array}$ & $\begin{array}{c}\text { Primary } \\
\text { level }\end{array}$ & $\begin{array}{c}\text { Middle } \\
\text { level }\end{array}$ & $\begin{array}{c}\text { High } \\
\text { level }\end{array}$ \\
\hline Type 1 & $75 \%$ & $90 \%$ & $95 \%$ \\
\hline Type 2 & $20 \%$ & $45 \%$ & $70 \%$ \\
\hline Type 3 & $60 \%$ & $60 \%$ & $95 \%$ \\
\hline Type 4 & $40 \%$ & $50 \%$ & $90 \%$ \\
\hline Type 5 & 0 & $5 \%$ & $30 \%$ \\
\hline
\end{tabular}

If $70 \%$ of respondents in each group was used as the sign that most respondents have mastered the sentence structure and relevant speech processing strategies, the speech processing strategies of most learners of three Chinese proficiency levels can be judged as follows: most primarylevel and middle-level learners are in the stage of "sequential combination strategy of verb-quantifiers", and most high-level learners are in the stage of "verb-object phrase separation and insertion strategy".

\section{CONCLUSION}

To learn Chinese quantifier complements, English speakers experience sequential combination strategy of verb- 
quantifiers < verb-object phrase adding strategy < verbobject phrase separation and insertion strategy < separable word separation and insertion strategy from the low level to the high level. The whole process of speech processing strategies reflects the development from external processing to internal processing, which is from external adding of components to internal separation and insertion of components. Internal separation of component involves the development from separation of different components to separation of single component. Some studies conclude that speech processing strategies for the second language learners and their development process also reflect development from external processing to internal processing, such as the development process of "adverb preposing < verb separation" in learning German as the second language and the development process of "combination < separation" in learning Chinese separable words as the second language. Will all second language learners experience the development process from external processing to internal processing during SLA? This is a research topic that deserves comprehensive analysis and deep studies.

\section{ACKNOWLEDGMENT}

This research was financially supported by National Social Science Foundation "16BYY100" and Education and Teaching Reform Project of Beijing Language and Culture University "AI201708”.

\section{REFERENCES}

[1] M. Pienemann, M. Johnston and G. Brindley, "Constructing an acquisition-based procedure for assessing second language acquisition", Studies in Second Language Acquisition, vol. 2, pp. 217-243, 1988.

[2] A. Mackey and J. Philp, "Conversational interaction and second language development: Recasts, responses and red herrings?", The Modern Language Journal, vol. 3, pp. 338-356, 1998.

[3] K. Yoshioka and T. Doi, "Testing the PienemannJohnston model with Japanese: a speech-processing view of the acquisition of particles and word order", Paper presented at the 8th Second Language Research Forum, Hawaii: University of Hawaii, 1988.

[4] Shuzi Sun, "Study on the Speech Processing Strategies of the Acquisition of Chinese Disyllables VO-Type Detachable Words", Master dissertation of Beijing Language and Culture University, 2004. (In Chinese).

[5] Ruifeng Wang, Comparative Study on the Speech Processing Strategies of Bi-Sentences of English Native Speakers and Japanese Native Speakers, Beijing: Beijing Language and Culture University Press, 2014. (In Chinese).

[6] Dexi Zhu, Grammar Notes, Beijing: Commercial Press, 1982. (In Chinese).

[7] Borong Huang, Xudong Liao, Morden Chinese, Beijng: Higher Education Press, 2003. (In Chinese).

[8] Ruanshiqinghe, Study on the Acquisition and Teaching Countermeasures of Chinese Quantitative Complement for Vietnamese Students, Doctoral dissertation of Liaoning University, 2016. (In Chinese).

[9] Minghuan Sun, A Study on the Errors Complementary Language in Korean Students, Doctoral dissertation of Jilin University, 2017. (In Chinese).

[10] Xiqiang Xiao, Xiaowei Rui, A Study on the Acquisition of Chinese Quantitative Complementary Sentences by Foreign Students, Study on Chinese as a Foreign Language, 2009. (In Chinese). 